\title{
Relación entre conocimiento y actitud sobre la donación de órganos en estudiantes de Puebla
}

\author{
Relationship between knowledge and attitude about \\ organ donation in Puebla students
}

\author{
Jesús Marcial-Romero, ${ }^{*}$ Nora Marina Fernández-Tamayo, ${ }^{\ddagger}$ Martha Elena Barrientos-Núñez ${ }^{\S}$ \\ * Benemérita Universidad Autónoma de Puebla, Facultad de Medicina, Puebla, Puebla. Unidad de Trasplantes Puebla. Medicina General. \\ ₹ Benemérita Universidad Autónoma de Puebla, Facultad de Medicina, Puebla, Puebla. Departamento de Anatomía Patológica. \\ $\S$ Unidad de Trasplantes Puebla. Especialidad en Medicina Familiar.
}

\section{RESUMEN}

Introducción: Determinar si existe relación entre el nivel de conocimientos y actitud hacia la donación de órganos, mediante la encuesta como instrumento ya validado en estudiantes de la preparatoria Alfonso Calderón Moreno y la facultad de Medicina de la Benemérita Universidad Autónoma de Puebla (BUAP). Material y métodos: Estudio descriptivo, trasversal, observacional, cualitativo, cuantitativo comparativo aplicado a los estudiantes de las instituciones mencionadas que utilizó la validación del instrumento con estabilidad por test-retest y homogeneidad con Alfa de Cronbach como componentes de confiabilidad y validez con la comprobación de la hipótesis para un nivel de confiabilidad del 95\% con la prueba estadística t de Student, normalidad de Kolmogorov-Smirnov y la posterior lectura de p valor. Resultados: Se obtuvo la confiabilidad del instrumento a través del coeficiente de correlación de 0.96 , la cual incluye dos variantes fundamentales, conocimiento y actitud, un coeficiente Alfa de Cronbach de 0.83, con una prueba de normalidad de Kolmogorov-Smirnov de 0.057 y un valor por t de Student con un p valor de 0.0082 . Conclusión: El instrumento realizado muestra cualidades psicométricas aceptables en confiabilidad, y a su aplicación a nuestra muestra nos deja a evidencia la relación proporcional entre la variante conocimiento y la variante actitud en cuanto a la donación de órganos y tejidos con fines de trasplante.

Palabras clave: Trasplantes, obtención de tejidos y órganos, muerte encefálica, actitud, México.

\begin{abstract}
Introduction: To determine if there is a relationship between the level of knowledge and attitude towards organ donation, through the survey as an instrument already validated in students of the Alfonso Calderón Moreno High School and the Faculty of Medicine of the Benemérita Universidad Autónoma de Puebla (BUAP). Material and methods: Descriptive, cross-sectional, observational, qualitative, quantitative comparative study that used the validation of the instrument with stability by retest and homogeneity with Cronbach's alpha as components of reliability and validity with the testing of the hypothesis for a level of reliability of $95 \%$ with the statistical test $t$ of Student, normality of Kolmogorov-Smirnov and the subsequent reading of $p$ value. Results: The reliability of the instrument was obtained through the correlation coefficient of 0.96 which includes two fundamental variants, knowledge and attitude, a Cronbach's alpha coefficient of 0.83 , with a Kolmogorov-Smirnov normality test of 0.057 and a value per Student's $t$ with a p value of 0.0082. Conclusion: The instrument performed shows acceptable psychometric qualities in reliability, and its application to our sample reveals the proportional relationship between the variant knowledge and the variant attitude regarding organ and tissue donation for transplant purposes.
\end{abstract}

Keywords: Transplants, rissue and organ procurement, brain death, attitude, Mexico.

Citar como: Marcial-Romero J, Fernández-Tamayo NM, Barrientos-Núñez ME. Relación entre conocimiento y actitud sobre la donación de órganos en estudiantes de Puebla. Rev Mex Traspl. 2020; 9 (3): 101-108. https://dx.doi.org/10.35366/97458 


\section{INTRODUCCIÓN}

El trasplante de órganos y tejidos es el tratamiento de elección para una gran cantidad de pacientes que presentan insuficiencia aguda o crónica de algún órgano o tejido. Sin embargo, la escasez de órganos donados con fines de trasplante es un grave problema en todo el mundo. ${ }^{1}$

En México, se tienen documentados los primeros trasplantes de riñón y de córnea en 1963, luego le siguieron los de hígado, páncreas, corazón, médula ósea y más recientemente los de células y tejidos, cuyo potencial ha creado grandes expectativas. En 1984, se publica la Ley General de Salud, en donde se incluye el título decimocuarto, denominado «Disposición de Órganos, Tejidos, Células y Cadáveres de seres humanos». Tres años después, se reforma la Ley General de Salud y se sientan las bases para la realización de trasplantes, además se crea el Programa y el Registro Nacional de Trasplantes. ${ }^{2}$

El 26 de mayo de 2000 se publica el decreto por el que se reforma el Título Decimocuarto de la Ley General de Salud sobre donación, trasplantes y pérdida de la vida. En ese año, se crea y delega al Centro Nacional de Trasplantes (CENATRA) el Registro Nacional de Trasplantes y se estableció que, junto con los Centros Estatales de Trasplantes (COETRAS), decidirá y vigilará la asignación de órganos, tejidos y células. La reforma mencionada introduce además cambios importantes en cuanto a la estructura hospitalaria en relación con los profesionales dedicados a los trasplantes, así como la figura de un responsable sanitario en los establecimientos y la del Comité Interno de Trasplantes. Se modifica también el sistema de autorización expresa, por escrito y tácita por medio de la figura del Disponente Secundario. ${ }^{3}$

La investigación realizada en Madrid, España sobre los aspectos psicosociales en la donación de órganos muestran que la falta de conocimiento del proceso de donación impide que las personas se comprometan a convertirse en donadores, en específico se identificó que hay poco conocimiento del tema, existen miedos y tabúes, principalmente biológicos y emotivos, que impactan de manera negativa en la donación de órganos y que la mayoría de las personas tienen una actitud positiva hacia la donación de órganos. ${ }^{4}$

En un trabajo realizado en Chile acerca de donación de órganos, compresión y significado, se concluye lo siguiente: el tema de la donación de órganos es desconocido; las personas dudan de la honorabilidad y talento ético de uno de los principales actores en este proceso, «los doctores»; hay un desconocimiento significativo de lo que es muerte encefálica, y su centralidad en la donación de órganos; la imagen de muerte que predomina en el común de las personas es el cese de la función cardiaca y respiratoria; la imagen de las personas encargadas de preguntar a los familiares respecto a la donación no es buena; el cuerpo cadavérico goza de un estatus especial; y más que hablar de altruismo, es preferible hablar de acciones prosociales. ${ }^{5}$

En el trabajo sobre los factores sociales y psicológicos que influyen en la donación de órganos realizado en Sevilla, España, se encontró que el sexo no influye en la actitud hacia la donación, los jóvenes entre 16 y 30 años tienen una actitud más positiva, en contraste con los mayores de 50 y menores de 16 que manifiestan una actitud negativa, las personas con un alto nivel educativo tienen actitudes positivas y a medida que el nivel cultural desciende, la predisposición a donar órganos es menor, además, las personas con ingresos más altos tienen una actitud más favorable. ${ }^{6}$

Tabla 1: Datos generales.

\begin{tabular}{|c|c|c|c|}
\hline Variable & Valor & $n$ & $\%$ \\
\hline \multirow[t]{10}{*}{ Edad } & 15 & 45 & 11.25 \\
\hline & 16 & 43 & 10.75 \\
\hline & 17 & 90 & 22.50 \\
\hline & 18 & 20 & 5.00 \\
\hline & 19 & 58 & 14.50 \\
\hline & 20 & 34 & 8.50 \\
\hline & 21 & 31 & 7.75 \\
\hline & 22 & 20 & 5.00 \\
\hline & 23 & 30 & 7.50 \\
\hline & 24 & 29 & 7.25 \\
\hline \multirow[t]{2}{*}{ Género } & Masculino & 172 & 43.00 \\
\hline & Femenino & 228 & 57.00 \\
\hline \multirow[t]{9}{*}{ Religión } & Agnóstico & 5 & 1.25 \\
\hline & Católico & 272 & 68.00 \\
\hline & Creyente & 1 & 0.25 \\
\hline & Cristiano & 35 & 8.75 \\
\hline & Evangélico & 2 & 0.50 \\
\hline & Judaísmo & 1 & 0.25 \\
\hline & Laveyano & 2 & 0.50 \\
\hline & Testigo de & 1 & 0.25 \\
\hline & $\begin{array}{l}\text { No especifi- } \\
\text { cado }\end{array}$ & 81 & 20.25 \\
\hline \multirow[t]{3}{*}{ Procedencia } & Costa & 12 & 3.00 \\
\hline & Sierra & 23 & 5.75 \\
\hline & Ciudad & 365 & 91.25 \\
\hline
\end{tabular}

Fuente: Elaboración propia. 


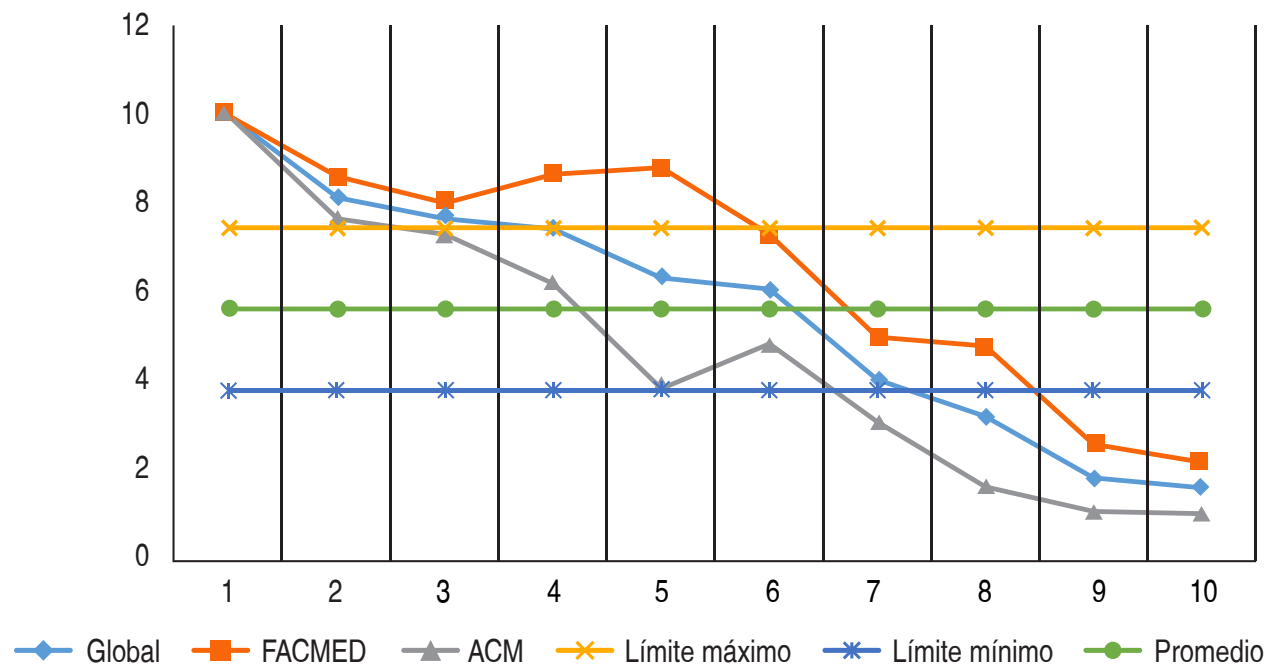

Figura 1:

De las 400 encuestas realizadas, se observa una gráfica decreciente en el conocimiento, principalmente en el ámbito legal y el órgano rector de la donación en México.

FACMED: Facultad de Medicina. ACM: Alfonso Calderón Moreno. Fuente elaboración propia.
En el estudio de Dueñas $C$ se analizan los factores asociados a la cultura de la donación en el Hospital General de Pachuca, Hidalgo y se llega a la conclusión de que no existe la cultura de donación de órganos, principalmente por falta de información clara y precisa desde temprana edad, porque se desconoce la forma en que se trata al donante durante la extracción, la población no identifica cuáles instituciones brindan información y orientación sobre donación y que existen sentimientos encontrados por el duelo y los mitos (como la mutilación, el tráfico de órganos y la posición de la Iglesia). ${ }^{7}$

La probabilidad de que una persona se convierta en un donador multiorgánico es muy baja, aunque esté de acuerdo en donar sus órganos al morir. Primero, es necesario que la persona fallezca en condiciones de muerte encefálica, dentro de una institución médica que cuente con licencia de procuración de órganos emitida por COFEPRIS. De acuerdo con el Consejo Estatal de Trasplantes de Jalisco, las estadísticas internacionales indican que los casos de muerte encefálica son de entre 50 y 80 personas por cada millón de habitantes. ${ }^{8}$

La estadística en la República Mexicana es desalentadora a comparación de los países con alta tasa de donación. ${ }^{9}$ El último informe anual dado por el Centro Nacional de Trasplante (CENATRA) corte al 03 de enero de 2020 de donación y trasplantes muestra los siguientes datos: de los receptores en espera se tienen 17,069 riñón, 5,713 córnea, 317 hígado, 43 corazón siete riñón-riñón, cuatro pulmón, tres hígado-riñón y dos riñón-páncreas.
El total de donaciones concretadas de personas fallecidas en México está en aumento, principalmente con el diagnóstico de paro cardiaco irreversible, el cual en 2007 fue de 508 mientras que en 2019 fue de 1,928. Por otra parte, el aumento del diagnóstico de muerte encefálica no ha sido tan significativo como el anterior, teniendo en 2007 una donación de 358, y en 2019 un total de 565 donaciones concretadas. ${ }^{9}$

Se debe hacer mención especial que el estado de Puebla, de acuerdo a lo graficado por el CENATRA en 2019, ocupa el lugar número 3 de donaciones en general, el número 4 de donación por muerte encefálica con 32 donaciones y el número 5 por paro cardiaco con 84 donaciones. ${ }^{9}$

Además de los factores contextuales y de los referidos al comportamiento de los profesionales sanitarios, la voluntad expresada en vida sobre la donación por parte de la persona fallecida y las disposiciones hacia la donación de los familiares participantes en el proceso de decisión son, entre otros, elementos cruciales que configuran la postura final sobre la donación. ${ }^{10}$

Adicional al trabajo educativo desarrollado con el personal vinculado a la coordinación de donación de órganos, parece evidente que deberían realizarse intervenciones dirigidas a la población general con la finalidad última de convertir la donación de órganos en una conducta habitual dentro de nuestra sociedad. El diseño de intervenciones educativas que tengan por objeto la mejora del clima social sobre donación y trasplantes debe fundamentarse en el previo conocimiento de la percepción social de la población sobre la que se desea intervenir. 
En la ciudad de Puebla, la falta de donación de órganos y tejidos contribuye un reto a las prácticas médicas ya que muchos tratamientos necesitan forzosamente un trasplante, más aún el aumento de la lista de espera del CENATRA para este año es de 23,405 y la negativa a la donación se mantiene en la población general, estudios ya realizados en diferentes países presentan una asociación entre la aceptación a la donación y el nivel de estudios que se tenga; sin embargo, en la ciudad de Puebla los estudios de este tipo

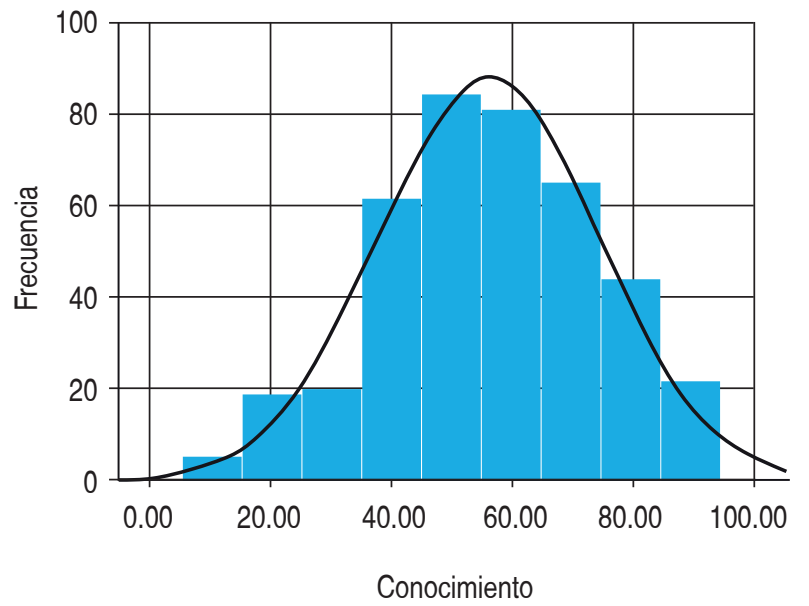

Media $=56.30 \quad$ Desviación estándar $=18.197 \quad \mathrm{~N}=400$

Figura 2: Se puede observar una asimetría negativa de -0.190 , indicando que las frecuencias mayores se ubican a la derecha de la gráfica, sin embargo, de manera discreta. La curtosis fue de -0.365 .

Fuente elaboración propia IBM SPSS. son escasos. En la actualidad, se emplean técnicas para educar a la comunidad a la donación cadavérica, aunque el alcance de ésta no ha sido el suficiente para disminuir de manera significativa la lista de espera para un trasplante.

Objetivo: determinar si existe relación entre el nivel de conocimientos y actitud hacia la donación de órganos, mediante la encuesta como instrumento ya validado en estudiantes de la preparatoria Alfonso Calderón Moreno y la facultad de Medicina de la Benemérita Universidad Autónoma de Puebla (BUAP).

\section{MATERIAL Y MÉTODOS}

Se trató de un estudio descriptivo, trasversal, observacional, cualitativo, cuantitativo, comparativo. El estudio se realizó en la preparatoria Alfonso Calderón Moreno de la Benemérita Universidad Autónoma de Puebla y la Facultad de Medicina de la misma institución en el periodo enero-mayo 2020. La población fue de ambos sexos de edad entre 15 y 24 años, que en el momento de realizarse la encuesta se encontraron en educación media superior o superior, conformada por 400 estudiantes de los cuales 200 pertenecían a educación media superior y 200 a educación superior de la BUAP. Se utilizó el método de muestreo aleatorio simple para un nivel de confianza de 95 y $5 \%$ de precisión, a 100\% de respuesta. La muestra se determinó con base en los datos del censo de población y vivienda 2015 en donde se contabilizaron en la ciudad de Puebla 1,161,001 habitantes con edad mayor o igual de 15 años y menores de 25 años. Se obtuvo como
Varianza comparativa conocimiento

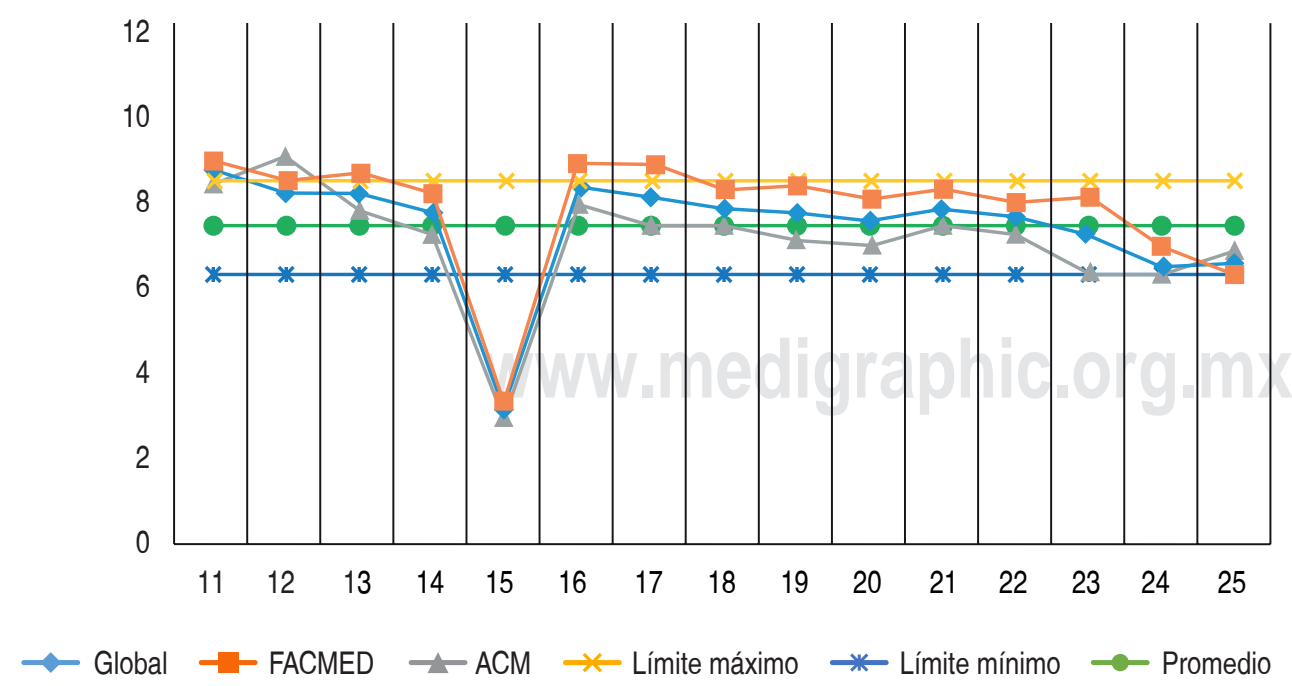

Figura 3:

La actitud que tiene nuestra muestra es en general a favor de ésta, teniendo una distribución normal entre los participantes. FACMED: Facultad de Medicina. ACM: Alfonso Calderón Moreno. Fuente elaboración propia. 


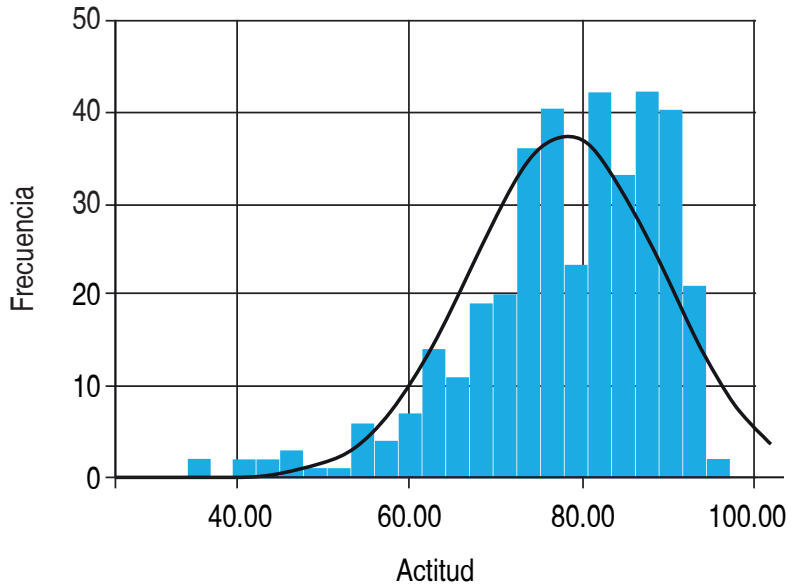

Media $=56.30$

Desviación estándar $=18.197$

$\mathrm{N}=400$

Figura 4: Se puede observar una asimetría negativa de -0.991, lo que indica que las frecuencias mayores se ubican a la derecha de la gráfica, con una tendencia muy marcada. La curtosis fue de 1.142 indicando una tendencia a la media.

Fuente elaboración propia IBM SPSS.

muestra óptima $n=399.86223$; por lo cual, se procedió a recopilar la información basada en 400 personas mayores de 15 años y menores de 24 años de la ciudad, elegidas de acuerdo a los criterios de inclusión. Se utilizó Software Microsoft Office Professional Plus 2013 Excel además de IBM SPSS Statistics Software.

La confiabilidad del instrumento se valoró por su estabilidad y homogeneidad, y la validez se midió con una $p<0.05$ donde antes se midió la normalidad de la muestra.

\section{RESULTADOS}

La población estudiada se eligió con base en diversos estudios que demuestran que las personas en edades jóvenes de 15 a 24 años tienen una mejor predisposición a la donación de órganos y tejidos con fines de trasplante.

Para lograr la investigación, se desarrolló un instrumento capaz de analizar las variables conocimiento y donación, además de determinar la confiabilidad y validez de constructo del instrumento. Se realizó una descripción general de los datos arrojados por el instrumento que, aunque no hace parte de la metodología para hallar la confiabilidad, hace parte de la responsabilidad social destacar los datos más relevantes que pueden concordar con la teoría recopilada hasta el momento o poner la alerta frente a situaciones que hasta ahora no han sido abordadas con el debido esmero.
Del $100 \%$ de encuestas incluidas se reportó: $11 \%$ en edad de 15 años, $11 \%$ en edad de 16 años, $22 \%$ en edad de 17 años, 5\% en edad de 18 años, 14\% en edad de 19 años, $9 \%$ en edad de 20 años, $8 \%$ en edad de 21 años, $5 \%$ en edad de 22 años, $8 \%$ en edad de 23 años y un 7\% en edad de 24 años. Del 100\% de las encuestas realizadas se tuvo un predominio del sexo femenino con un $57 \%$ en contra del género masculino con un $43 \%$. La religión de los encuestados fue en su mayoría católica con un $68 \%$. Se realizó el instrumento con habitantes de la ciudad en un $91 \%$, esto para no afectar la propia estadística del estudio por la diversidad que existe. Se encontró un $6 \%$ de pertenecientes a sierra y un $3 \%$ pertenecientes a costa (Tabla 1).

En los 400 encuestados, el conocimiento tiene diferentes variantes, aunque principalmente se encuentran en la media, hay claras diferencias. Los estudiantes definen correctamente lo que es donación, lo cual nos da un valor alto de conocimiento con un porcentaje de la muestra del $99 \%$. El reconocimiento de los tipos de donantes es alto, al igual que las condiciones y contraindicaciones para ser donante, esto es bastante importante y relevante, ya que se puede intuir que la población está enterada de estos procesos; lo anterior contrasta con el pensamiento colectivo de que las personas desconocen esto y que por esta razón no aceptan ser donadores, estos porcentajes los encontramos en un valor de 77 y $60 \%$ respectivamente. Sin embargo, encontramos un conocimiento bajo en la importancia de la donación de órganos donde no se

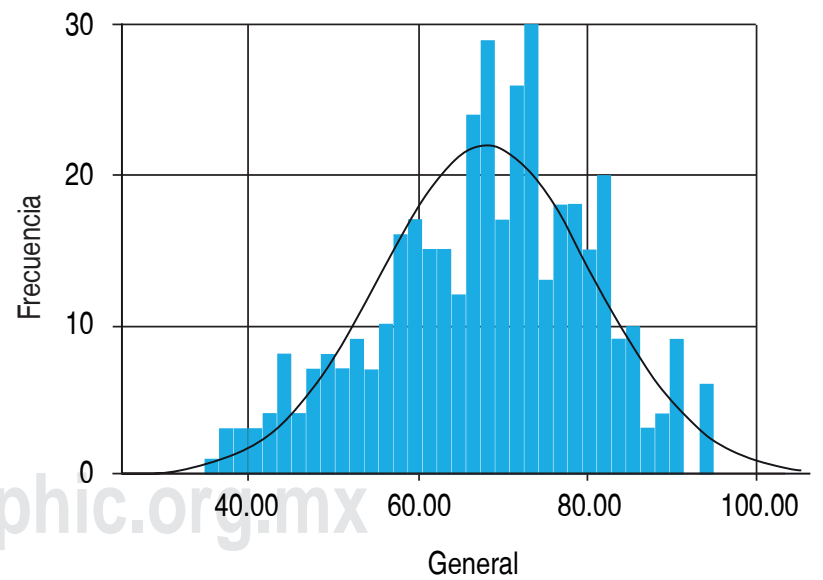

Media $=56.30$

Desviación estándar $=18.197$

$N=400$

Figura 5: Se puede observar una asimetría de negativa en -0.321, indicando que las frecuencias mayores se ubican a la derecha de la gráfica. La curtosis fue de -0.327 .

Fuente elaboración propia IBM SPSS. 
conoce el número de personas que ayuda una donación y en menor cantidad se ignora el número de pacientes en espera de un trasplante con un porcentaje del $38.5 \%$. Por último, tenemos el marco legal donde la población muestra, en la que se aplicó el instrumento, presenta un conocimiento bajo con un porcentaje de $25 \%$, cabe mencionar que las preguntas del marco legal no incluyen artículos, sólo el conocimiento de la ley vigente sobre la donación y el trasplante en México y el órgano encargado de esto (Figura 1).

Se puede observar una asimetría negativa de -0.190 que indica que las frecuencias mayores se ubican a la derecha de la gráfica, sin embargo, de manera discreta la curtosis fue de -0.365 (Figura 2).

La actitud que tiene nuestra muestra es en general a favor de ésta, teniendo una distribución normal entre los participantes. Las percepciones, creencias y la información que tiene acerca de la donación de órganos y tejidos es de aceptación; sin embargo, en este apartado se encuentra la pregunta sobre su percepción hacia el tráfico de órganos, a lo cual la mayoría considera que este existe en el país, esto puede estar relacionado con la falta de conocimiento de la legislación aplicable para la donación de órganos y tejidos. Las sensaciones y sentimientos son de aceptación en los estudiantes en su mayoría. Por último, las intenciones, disposiciones o tendencias del estudiante hacia la donación de órganos son de aceptación; sin embargo, no encontramos una muestra con tendencia a respuesta positiva en general, sino varianzas entre los límites del ítem principalmente en la participación activa y la disposición para convertirse en futuros donadores altruistas. Por otra parte, un punto destacable es que la mayoría de los encuestados tienen una actitud muy favorable a recibir capacitación sobre el tema de donación (Figura 3). Se puede observar una asimetría negativa de -0.991 , lo cual indica que las frecuencias mayores se ubican a la derecha de la gráfica, con una tendencia muy marcada. La curtosis fue de 1.142, indicando una tendencia a la media (Figura 4).

Se observa una tendencia a la normalidad de las respuestas de los participantes. Se encontró que el valor promedio fue de 67.82 , teniendo como puntaje máximo posible 93, límite superior 69, límite mínimo 66.6 y mínimo 36. Respecto a la dispersión de los datos, la desviación estándar es de 12. Se puede observar una asimetría de negativa en -0.321, que indica que las frecuencias mayores se ubican a la derecha de la gráfica. La curtosis fue de -0.327 (Figura 5).

Hallar la confiabilidad y la validez de un instrumento de medida es un paso imprescindible en este cami- no del conocimiento que permite la utilización en la comunidad científica, para esto se utilizó la confiabilidad test-retest como técnica para conocer la estabilidad de la prueba en dos momentos diferentes, a través del coeficiente de Pearson se encontró una confiabilidad de 0.96 en el instrumento en general que demuestra una estabilidad deseable y adecuada, permitiendo afirmar que el test proporciona medidas precisas, fiables, dignas de confianza. La validez se realizó con el cálculo de la consistencia interna utilizando el coeficiente Alfa de Cronbach, que para la escala en general reportó un Alfa de 0.83 , lo que es aceptable, permitiendo afirmar que en general la escala es homogénea. Por último, cabe recordar que el test es un instrumento de medida y diagnóstico y su eficacia depende tanto de su validez y precisión como de la competencia y pericia del que lo usa. Todo test, por exacto y válido que sea, refleja sólo un aspecto parcial de la personalidad del sujeto en un momento arbitrariamente fijado de su vida. ${ }^{11-13}$

La posterior aplicación del instrumento a la muestra establecida nos concluye que la hipótesis planteada es correcta y que a mayor nivel de conocimiento mayor actitud hacia la donación de órganos y tejidos con fines de trasplante.

\section{DISCUSIÓN}

En la actualidad, el trasplante constituye uno de los grandes avances terapéuticos, sin embargo, éste se ve frenado por la falta de donación de la población. Diversos estudios demostraron que la actitud hacia la donación se ve influenciada principalmente por el nivel de conocimiento, no sólo a nivel global, sino también internamente en la República Mexicana tenemos estos resultados, aunque son escasos estos estudios, aún más en el estado de Puebla donde no existen estudios que observen la actitud de la población hacia la donación.

La variable de interés es la relación entre el nivel de conocimiento y la actitud hacia la donación de órganos y tejidos con fines de trasplante en las que no se ha profundizado lo suficiente.

El principal aporte del estudio es conocer la relación entre el conocimiento y la actitud hacia la donación en una muestra con criterios de edad menores de 25 años, en la que diversos autores han concluido que tienen una buena actitud. Ésta se dividió en dos grupos: uno que por sí mismo tiene mayor conocimiento al estar en el área médica y el segundo con un conocimiento intermedio, similar al de la población habitual no relacionada al área de la salud. Ya que en 
el estado de Puebla no existe documentación sobre este tipo de estudios, se construyó un instrumento capaz de dar a conocer estas dos variables y si tienen alguna relación.

Esto nos presenta el escenario de diversas referencias consultadas, donde la falta de conocimiento sobre la legislación provoca desconfianza y con ello que el número de donantes no aumente por miedo a la mutilación del cuerpo.

Los datos correspondientes a la actitud hacia la donación dieron una aceptación general en las tres áreas valoradas, cognitiva, afectiva y conductual. Sin embargo, el estudio demuestra en el Área Cognitiva que la muestra opina que existe el tráfico de órganos, esto tiene una relación proporcional con la falta de conocimiento de la legislación y el órgano encargado de todo el procedimiento de donación y trasplante, incluida su distribución y trazabilidad.

Se deben tomar con discreción los resultados, ya que aunque la muestra fue lo suficientemente grande para arrojar resultados congruentes, ésta se ve influenciada por otras variables como son edad, religión y procedencia. Asimismo, a pesar de que la hipótesis se comprobó, es decir, el conocimiento es proporcional a la actitud sobre la donación, la Facultad de Medicina se encuentra más en contra de la donación comparada con la preparatoria, esta última se encuentra más indiferente al tema que la propia Facultad. Se debe tener en cuenta que las universidades no tienen un programa de donación, incluida la Facultad de Medicina, y pocas materias tocan este tema además de que los estudios y la información actualizada sobre donación son escasos.

\section{CONCLUSIONES}

El trabajo realizado nos deja al manifiesto las conclusiones que diversos artículos comentan, donde esta relación se ve poco influenciada por el género y que su relación del conocimiento va directamente relacionada con la actitud. Es importante trabajar en este hecho fundamental con capacitación continua a nuestra población sobre donación altruista y con mayor énfasis en la población joven, que ya se ha demostrado su buena actitud hacia este tema, pero su conocimiento es intermedio, sobre todo trabajar el aspecto legal de este componente. La observación de los médicos en formación, aunque demuestra un mayor conocimiento y actitud, también demuestra su desconocimiento sobre la legislación, lo cual es preocupante al no conocer este apartado tan importante, sobre todo porque el médico es fundamental para la aceptación o rechazo de la donación de órganos y tejidos.

Dentro de las limitaciones encontradas en el estudio, se observó una falta de diversidad en cuanto a la religión y su procedencia ya que la mayoría mostró ser católico y de ciudad, para concluir si estas variables repercuten directamente en la actitud hacia la donación se deberían hacer más estudios comparativos entre diferentes poblaciones pertenecientes a estas variantes.

\section{REFERENCIAS}

1. Vázquez M, Moreno S. Donación de órganos y tejidos con fines de trasplante, lo que el médico en formación debe conocer. Hospital General Regional Núm. 1, Instituto Mexicano del Seguro Social, Michoacán, México. Rev Mex Traspl. 2018; 7 (2): 59-64. [Consultado 14 jul 2020]. Disponible en: https://www. medigraphic.com/pdfs/trasplantes/rmt-2018/rmt182d.pdf.

2. Ramírez I. Aspectos médicos, éticos y jurídicos sobre trasplantes de órganos y tejidos. En: Temas selectos de salud y derecho. Instituto de Investigaciones Jurídicas. Universidad Autónoma de México. México D.F. 2002. pp. 109-118 [Consultado 25 mar 2020]. Disponible en: http://biblio.juridicas.unam.mx/ libros/1/357/9.pdf.

3. Dib A, Aburto S, Espinosa A, Sánchez O. Trasplante de órganos en México. Centro Nacional de Trasplantes. Secretaría de Salud. Rev Invest Clin 2005; 57 (2): 163-169. [Consultado 25 mar 2020]. Disponible en: http://www.scielo.org.mx/pdf/ric/ v57n2/v57n2a9.pdf.

4. González A, Martínez J, Manrique S. Donación de órganos para trasplantes, aspectos psicosociales. Madrid, España. Universidad Autónoma de Madrid. Nefrología. 1991; 11 (Supl. 1): 62-68.

5. Guerra R. Donación de órganos comprensión y significado. [Tesis para obtener el título de licenciado en sociología]. Chile. Universidad de Chile; 2005.

6. Pérez M, Domínguez J, Murillo F. Factores sociales y psicológicos que influyen en la donación de órganos. Psicothema. 1993; 5 (2): 241-253.

7. Dueñas C. La cultura de la donación de órganos y tejidos en el Hospital General de Pachuca. México. Universidad Autónoma del Estado de Hidalgo. Área Académica de Trabajo Social. [Tesis para obtener el título de licenciado en trabajo social]. Hidalgo, México. 2005.

8. Consejo Estatal de Trasplantes de Jalisco. El donante. Muerte Encefálica. [Internet] Jalisco. [Consultado 16 abr 2020]. Disponible en: http://app.jalisco.gob.mx/Trasplantes.nsf/3c2 a1d46742d325186256fc500689382/7fd626e9ddb2b3df862 578ca005ce296/\$FILE/DONANTE\%20MUERTE\%20ENCEFALICA\%202011.pdf.

9. Centro Nacional de Trasplantes. Reporte Anual 2019 de donación y trasplantes en México [Internet]. Sistema Informático del Registro Nacional de Trasplante. [Actualizado 03 ene 2020] [Consultado 25 mar 2020]. Disponible en: https://www.gob.mx/cms/uploads/ attachment/file/528301/Presentacion_anual_2019.pdf.

10. Instituto Nacional de Salud. Red de donación y trasplante de órganos y tejidos [Internet]. Instituto Nacional de Salud. Bogotá. Colombia. 2017 [Consultado 16 abr 2020]. Disponible en: http://www.ins.gov.co/ lineas-de-accion/red-nacional-laboratorios/paginas/red-dedonaciony-trasplante-de-organos-y-tejidos.aspx\#. WIAqSVPhDIU. 
11. Radecki C, Jaccard J. Psychological aspects of organ donation: a critical review and synthesis of individual and next-of-kin donation decisions. Health Psychol. 1997; 16 (2): 183-195.

12. Magnusson D. Teoría de los tests. México. 2 ed. Editorial Trillas, México, 2009.

13. Sánchez R, Echeverria J. Validación de escalas de medición en salud [Internet]. Revista En: Salud pública. [Actualizado 2004]
[Consultado 16 abr 2020]. Disponible en: https://www.scielosp. org/article/rsap/2004.v6n3/302-318/.

Correspondencia: Jesús Marcial-Romero

E-mail: susej300195@gmail.com 